\title{
Stereotactic Body Radiation Therapy (SBRT) for Liver Oligometastases: Outcomes and Safety
}

\author{
Dejan Cazic ${ }^{1}$, Goran Marosevic ${ }^{1,2}$ \\ ${ }^{1}$ International Medical Centers Banja Luka, Affidea, Center for Radiotherapy, Banja Luka, Bosnia and Herzegovina, ${ }^{2}$ Medical \\ Faculty, University of Banja Luka, Banja Luka, Bosnia and Herzegovina
}

Correspondence: dejan.cazic@affidea.com; Tel.: + 38765871 984; Fax.: + 38751340510

Received: 13 July 2020; Accepted: 30 December 2020

\begin{abstract}
Objective. The aim of this study was to investigate adverse effects, progression free survival (PFS), one-year local control (LC) and one-year overall survival (OS) of patients with liver oligometastases treated with stereotactic body radiotherapy (SBRT), and whether there was a significant difference in these parameters in patients with primary colorectal cancer compared to other tumor localizations. Patients and Methods. Patients were simulated using four-dimensional computed tomography (4DCT). Using volumetric modulated arc therapy (VMAT) technique, SBRT was performed on 16 patients with $<3$ liver metastases. The prescribed dose was $60 \mathrm{~Gy}$ in 8 fractions (BED $105 \mathrm{~Gy}$ ). Cone beam CT (CBCT) was used for image guidance before each fraction with online correction. Results. There were no adverse effects. Median PFS for all patients, patients with primary colorectal cancer, and patients with primary non-colorectal cancer was 11 months (SE 2.1), 16 months (SE 2.8), 6 months (SE 2.4), respectively. There was no significant difference in the PFS for these two observed groups $(\mathrm{P}=0.09)$. The one-year LC was $62.5 \%$. Patients with primary colorectal cancer had one-year LC of $87.5 \%$, while the group of patients with primary non-colorectal cancer had one-year LC of $37.5 \%(\mathrm{P}=0.063)$. The total one-year OS was $87.5 \%$. In the group of patients with primary colorectal cancer, the one-year OS was $100 \%$, while in the group of patients with primary non-colorectal cancer, the one-year OS was $75 \%$ $(\mathrm{P}=0.317)$. Conclusion. SBRT with $8 \times 7.5 \mathrm{~Gy}$ can be safely delivered and is effective method of treating liver oligometastases.
\end{abstract}

Key Words: SBRT • Liver • Oligometastases.

\section{Introduction}

Metastases in the liver cause significant morbidity, causing pain and anorexia, among others, while significant liver dysfunction worsens general condition of the patient and is one of significant causes of mortality in patients with malignant diseases (1). Liver is one of the most common sites for metastatic spread. The largest number of liver metastases are of colorectal cancer origin (2, 3). Although it is a metastatic disease, if it is limited in number and localization only to the liver, these patients are candidates for local therapy (4, 5). Surgery is the first option for local treatment, with long-term good results in the control of oligometastatic liver disease (6-8). Unfortunately, only $10-20 \%$ of metastases in the liver are resectable, which is why most patients are treated with systemic therapy. Although new combinations of chemotherapeutic agents and targeted drugs lead to better results, they do not do so without significant toxicity. Other methods of local ablative therapies that have shown benefit are stereotactic body radiotherapy (SBRT), radiofrequency and microwave ablation, transarterial chemoembolization, cryoablation and alcohol injection (9-11). Radiotherapy is a proven palliative treatment method, and in patients with painful metastases in the liver, even one fraction of irradiation of the whole liver could achieve a significant reduction in symptoms and lead to improved quality of life in most patients $(12,13)$. 
Due to the low tolerance of the liver parenchyma to radiation and high risk of damage to the healthy liver parenchyma, the standard form of external radiotherapy to a larger volume of liver tissue is not an option for treating liver metastases (RILD, Radiation Induced Liver Damage) (1416). Technological advances have improved planning systems and imaging methods which made it possible to apply high doses of radiation in a smaller number of fractions to a limited volume in the liver parenchyma while maximally sparing the surrounding healthy parenchyma (17-21). By increasing the dose that can be safely applied to the tumor, the ability to control the tumor also increases. Numerous studies have been investigating the possibilities of application, efficiency and safety of stereotactic radiation of liver metastases.

The aim of this study is to investigate adverse effects, period up to disease progression (PFS, Progression Free Survival), one-year local control (LC) and one-year overall survival (OS) of patients with liver oligometastases treated with SBRT, as well as whether there is a significant difference in these parameters in patients with primary colorectal cancer compared to other tumor localizations.

\section{Patients and Methods}

\section{Patients}

This retrospective study included patients who were treated consecutively in the period from $\mathrm{Au}$ gust 2016 to June 2019. Sample size was formed in the way that patients were censored at the time of analysis (June 2020), so each patient had a followup time of at least one year. Inclusion factors for SBRT of liver metastases were: liver metastases that were unresectable or medically inoperable due to comorbidities and which were verified by biopsy or CT / MR / PET imaging with an increase in tumor markers, $<3$ size metastases, size $\leq 6 \mathrm{~cm}$, stable primary tumor, good liver function, in good general condition (ECOG 0-2). Exclusion factors were observed and later confirmed, through diagnostic tests, disease progression at the time of CT simulation and crossing the dose constraints on healthy tissues.

\section{Methods}

Patients were simulated using four-dimensional computed tomography (4DCT, GE LightSpeed, 16 slice, slice thickness $1.25 \mathrm{~mm}$ ) in supine position using abdominal compression (Macromedics), with or without intravenous contrast (22). For more accurate visualization and delineation, available pre-therapeutic diagnostic tests were registered (contrast-enhanced CT, MR, PET/ $\mathrm{CT}$ ). The target volume was contoured at the following phases of 4DCT: fb (free breathing), 0, 50, 90 and Min-IP (Minimal Intensity Projection) in the ARIA radiotherapy system (Varian Medical Systems Inc, Palo Alto, CA, USA), with target volume position check on all available diagnostic and 4DCT simulation images. By combining the contours in the mentioned phases, ITV (Internal Tumor Volume) was defined, which was named iGTV, and then PTV with a margin of 3-5 mm. Organs at risk were contoured according to the RTOG atlas and included: liver (liver contoured on fb series and Avg (Average) series, oesophagus, small intestine, large intestine, stomach, kidneys, spinal cord, spinal cord PRV, ribs and skin.

The prescribed dose was $60 \mathrm{~Gy}$ in 8 fractions (7.5 Gy daily; BED $105 \mathrm{~Gy}, \alpha / \beta=10$ ), every other day. Planning was done using the Eclipse planning system, and the Acuros XB algorithm was used to calculate the dose. The two half-arc volumetric modulated arc therapy (VMAT) technique with a treatment couch rotation of $\pm 10^{\circ}$ was used (Figure 1). The treatment was performed on a Varian Clinac DHX linear accelerator. Before each fraction, cone beam CT (CBCT) and online verification and correction of the patient's position and metastasis or metastases were performed. Checking the position of the target volume was controlled so that liver contour on the obtained CBCT image overlapped with the liver contour obtained on the Avg series of simulation $4 \mathrm{DCT}$, which was considered a surrogate for the liver position during treatment if metastases were not clearly visible $(23,24)$.

Patients were monitored prospectively with control laboratory tests (blood count, liver biochemical tests) and radiological imaging. First control laboratory tests were 2 weeks after treat- 


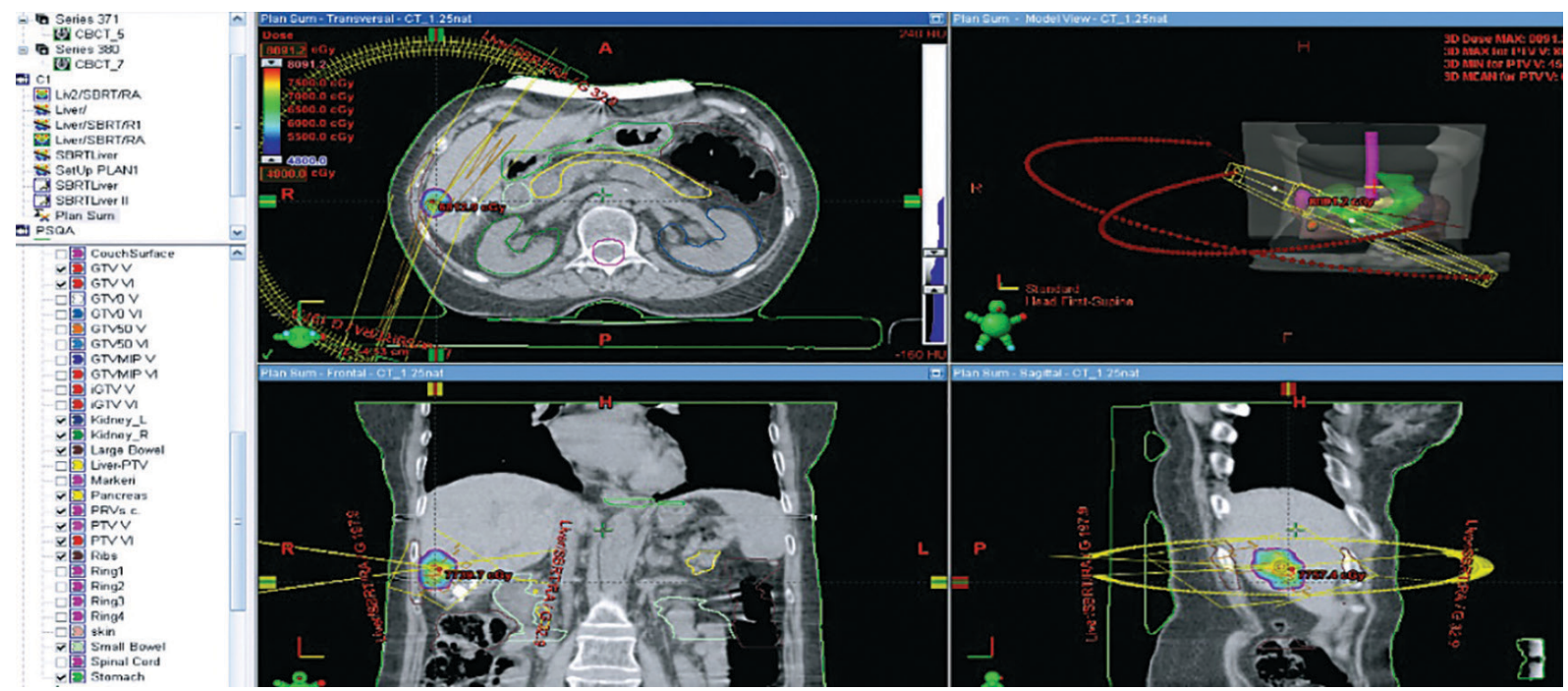

Figure 1. SBRT Plan for Liver Metastasis.

ment and then at quarterly intervals. Radiographic response assessment was performed quarterly according to RECIST 1.1 criteria and was classified as complete response (CR), partial response (PR), stable disease (SD) or progressive disease (PD). $\mathrm{CR}$ is defined as the total radiographic disappearance of all lesions. PR is defined as at least a 30\% reduction in the amount of diameter of the target lesions. PD is defined as at least a $20 \%$ increase in the diameter of the target lesions. In addition to a relative increase of $20 \%$, the lesion also had to show an absolute increase of at least $5 \mathrm{~mm}$. The appearance of one or more new lesions was also considered progression. SD is defined as neither a sufficient decrease to qualify for PR, nor a sufficient increase to qualify for PD (25).

Progression of the disease is considered to be the progression of the treated lesion, appearance of new metastases in the liver, distant organs and/or in the lymph nodes. One-year local control is met if the treated lesion is without signs of disease progression. Patients were followed for local control for up to a year in case of disease progression which was not a consequence of the progression of the treated lesion. In case the patient dies within one year from the beginning of the treatment, it was considered that the local control was not met. One-year overall survival was measured from the day of commencement of the stereotactic radiotherapy treatment.

\section{Statistical Analysis}

Standard descriptive methods (arithmetic mean with standard deviation, median with standard error, range of numerical feature from minimum to maximum value) were used in statistical data analysis. Survival analysis was performed using the Kaplan-Meier method. Survival curves were compared using the Log Rank test. The difference was considered significant if $\mathrm{P}<0.05$. IBM SPSS statistics software (ver. 1.0.0.1406) was used for statistical analysis.

\section{Results}

There were no treatment side effects or changes in blood counts and liver biochemical parameters. Number of eligible patients was 16 (6 male and 10 female patients), none met the criteria for exclusion. The mean age for the 16 patients analysed (Table 1) was 63 years (standard deviation 15.64, minimum 17 , maximum 78 ). The most numerous were the patients with primary colorectal cancer $(8$ patients). Four patients had primary breast cancer, 2 had primary cancer of the hepatobiliary tract, 1 had primary lung cancer and 1 had hepatocellular carcinoma. The total number of treated metastases was 21 . Ten patients received chemotherapy due to liver metastases before treatment with SBRT, while 
Acta Medica Academica 2020;49(3):225-231

Table 1. Patients Characteristics

\begin{tabular}{|c|c|c|c|}
\hline Characteristic & $\begin{array}{l}\text { All patients } \\
\mathrm{N}(\%)\end{array}$ & $\begin{array}{l}\text { Male } \\
\text { N (\%) }\end{array}$ & $\begin{array}{l}\text { Female } \\
\mathrm{N}(\%)\end{array}$ \\
\hline Number of patients & 16 & $6(37.5)$ & $10(62.5)$ \\
\hline \multicolumn{4}{|l|}{ Age (years) } \\
\hline$<65$ & $7(43.7)$ & $1(6.3)$ & $6(37.5)$ \\
\hline$\geq 65$ & $9(56.3)$ & $5(31.2)$ & $4(25)$ \\
\hline Median age & 63 (range 18-78) & 73 (range 62-78) & 56 (range 18-76) \\
\hline \multicolumn{4}{|l|}{ Primary tumor } \\
\hline Colorectal cancer & $8(50)$ & $5(31.2)$ & $3(18.8)$ \\
\hline Breast cancer & $4(25)$ & - & $4(25)$ \\
\hline Lung cancer & $1(6.3)$ & $1(6.3)$ & - \\
\hline Hepatobiliary tract cancer & $2(12.5)$ & - & $2(12.5)$ \\
\hline Primary liver cancer & $1(6.3)$ & - & $1(6.3)$ \\
\hline \multicolumn{4}{|c|}{ Number of liver metastases per patient } \\
\hline One & $11(68.8)$ & $3(18.8)$ & $8(50)$ \\
\hline Two & $5(31.3)$ & $3(18.8)$ & $2(12.5)$ \\
\hline \multicolumn{4}{|l|}{ Size of the metastases $(\mathrm{cm})$} \\
\hline$<2$ & 7 & - & - \\
\hline $2-4$ & 8 & - & - \\
\hline $4-6$ & 6 & - & - \\
\hline
\end{tabular}

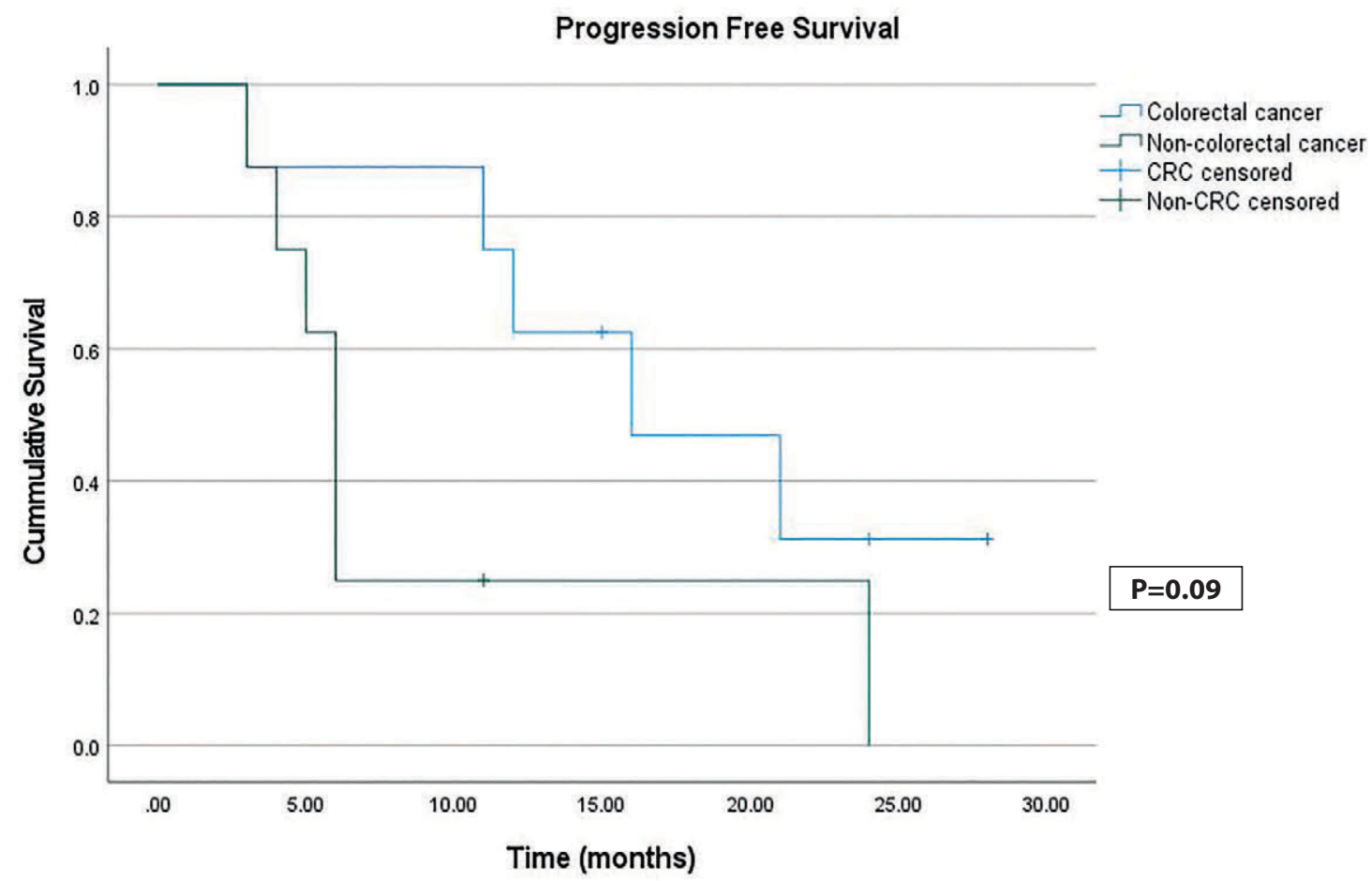

Figure 2. Progression Free Survival (PFS) Plot. 
in 6 patients SBRT was the first therapy for oligometastatic liver disease.

The median PFS for all patients was 11 months (standard error 2.1, minimum 3 months, maximum 28 months). In the group of patients with primary colorectal cancer, the median PFS was 16 months (standard error 2.8, minimum 3 months, maximum 28 months). In the group of patients with primary non-colorectal cancer, the median PFS was 6 months (standard error 2.4, minimum 3 months, maximum 24 months). There was no significant difference in the disease progression free survival for these two observed groups $(\mathrm{P}=0.09)$ (Figure 2). Sample mean follow-up time was 12.2 months (standard deviation 8.3).

The one-year LC was $62.5 \%$. In the group of patients with primary colorectal cancer, the oneyear LC was $87.5 \%$, while in the group of patients with primary non-colorectal cancer, the one-year LC was $37.5 \%$. There is no significant difference in one-year LC for patients with colorectal cancer compared to patients with primary tumor of other localizations $(\mathrm{P}=0.063)$. The total one-year OS was $87.5 \%$ (14 of 16 patients). In the group of patients with primary colorectal cancer, the oneyear OS was $100 \%$, while in the group of patients with primary non-colorectal cancer, the one-year OS was $75 \%$. There was no significant difference in the one-year OS for these two observed groups $(\mathrm{P}=0.317)$.

\section{Discussion}

This study investigates the safety and efficacy of SBRT in the treatment of patients with oligometastatic liver disease and it examines whether there is a difference in prognosis if the patient has primary colorectal cancer compared to other primary tumors. Compared to other studies (Rusthoven et al. PFS 6.1 months; Lee et al. PFS 3.9 months, Nicosia et al. PFS 7 months), an enviable median progression free survival has been achieved, especially for the group of patients with colorectal cancer (2628). Despite not having statistically significant difference in PFS for patients with colorectal cancer compared to other primary tumors $(\mathrm{P}=0.09)$, dif- ference in median time until disease progression is clinically significant (16 months vs. 6 months), in favour of patients with primary colorectal cancer.

The achieved one-year LC of $62.5 \%$ is slightly lower, while the one-year LC of $87.5 \%$ for patients with colorectal cancer is in line with the results of LC from other studies, ranging from $71 \%$ to $95 \%$ $(26,27,29-31)$. This study shows that there is no significant difference in LC in patients with colorectal cancer compared to other histologies $(87.5 \%$ vs $37.5 \%, \mathrm{P}=0.063)$. An explanation of the results of one-year LC can be found in the prescribed dose and size of treated metastases. The higher the dose, the greater the possibility of LC, as the study by Rule et al. shows that there is a significant difference in the two-year control between $30 \mathrm{~Gy}$ in 3 fractions and 60 Gy in 5 fractions ( $56 \%$ vs $100 \%, \mathrm{P}=0.009$ ) (32). One-year LC is $94 \%$ and $95 \%$ for the prescribed doses of $75 \mathrm{~Gy}$ in 3 fractions and $60 \mathrm{~Gy}$ in 3 fractions $(26,31)$. Dose escalation in these studies is safe in terms of tolerance and toxicity, if dose volume limits and liver volume are to be spared. According to the study by Rusthoven et al. 100\% two-year control was achieved for metastases of size $<3 \mathrm{~cm}$, while for metastases $>3 \mathrm{~cm}$ it was $77 \%$ $(\mathrm{P}=0.015)$ (26). In the group of patients this study analysed, only one third of patients had metastases $<2 \mathrm{~cm}$, while other metastases were $>2 \mathrm{~cm}$, which potentially affected somewhat lower total LC.

The one-year OS for all patients, patients with colorectal cancer, and patients with other primary tumors is $87.5 \%, 100 \%$, and $75 \%$, respectively. No significant difference in one-year survival is achieved between the observed groups $(\mathrm{P}=0.317)$. The results of other studies range from $68.6 \%$ to $83.5 \%$ (28-31). Rusthoven et al. showed that there was a group of favorable histologies, which included colorectal cancer, breast and kidney cancer, carcinoids, GIST and sarcomas, which had a significantly longer median survival of 32 months compared to other adverse histologies with a median survival of 12 months $(\mathrm{P}=0.001)$ (26). The difference in PFS and one-year LC shows that in patients with oligometastatic liver disease, primary colorectal cancer has a good prognosis, which is confirmed by the study of Andratschke et al. (29). 
The disadvantage of this study is the relatively small number of patients, which led to the formation of a group with combined patients with primary tumors other than colorectal cancer. That prevented individual comparison of outcomes between different primary tumors. The small sample also affected the result of PFS and one-year OS, i.e. the inability to obtain a significant difference between the observed groups.

\section{Conclusion}

In conclusion, patients treated with $8 \times 7.5 \mathrm{~Gy}$ SBRT experienced no treatment-related adverse effects. This dose-fractionation regime can be effective as a local ablative treatment of oligometastatic liver disease.

\section{What Is Already Known on this Topic:}

Liver metastases are significant cause of morbidity and mortality in patients with malignant diseases. Most patients are treated with systemic therapy. When these patients are candidates for local therapy, surgery is the first option. Stereotactic body radiotherapy (SBRT) shows benefit in patients who are not surgical candidates.

\section{What this Study Adds:}

This study showed that SBRT $(8 \times 7.5 \mathrm{~Gy})$ can be safely delivered and have positive effect in the treatment of liver metastases. We evaluated adverse effects, PFS, one-year local control and one-year overall survival of patients with liver oligometastases treated with SBRT. In addition, better prognosis for patients with colorectal cancer is observed.

Acknowledgements: The authors are grateful to Dijana Stricic for her help on English language review.

Authors' Contributions: Conception and design: DC and GM; Acquisition, analysis and interpretation of data: DC and GM; Drafting the article: DC and GM; Revising it critically for important intellectual content: DC and GM; Approved final version of the manuscript: DC and GM.

Conflict of Interest: The authors declare that they have no conflict of interest.

\section{References}

1. Costi R, Leonardi F, Zanoni D, Violi V, Roncoroni L. Palliative care and endstage colorectal cancer management: the surgeon meets the oncologist. World J Gastroenterol. 2014;20(24):7602-1. doi:10.3748/wjg.v20.i24.7602
2. Kemeny NE, Ron IG. Liver Metastases. Curr Treat Options Gastroenterol. 1999;2(1):49-57. doi:10.1007/s11938999-0018-1

3. Siegel RL, Miller KD, Jemal A. Cancer statistics, 2017. CA Cancer J Clin. 2017;67(1):7-30. doi:10.3322/caac.21387

4. Weiss L, Grundmann E, Torhorst J, Hartveit F, Moberg I, Eder M, et al. Haematogenous metastatic patterns in colonic carcinoma: an analysis of 1541 necropsies. J Pathol. 1986;150(3):195-203. doi:10.1002/path.1711500308

5. Hellman S, Weichselbaum RR. Oligometastases. J Clin Oncol. 1995;13(1):8-10. doi:10.1200/JCO.1995.13.1.8

6. Nordlinger B, Sorbye H, Glimelius B, Poston GJ, Schlag $\mathrm{PM}$, Rougier $\mathrm{P}$, et al. Perioperative FOLFOX4 chemotherapy and surgery versus surgery alone for resectable liver metastases from colorectal cancer (EORTC 40983): longterm results of a randomised, controlled, phase 3 trial. Lancet Oncol. 2013;14(12):1208-15. doi:10.1016/S14702045(13)70447-9

7. Smith JJ, Angelica D MI. Surgical management of hepatic metastases of colorectal cancer. Hematol Oncol Clin North Am. 2015;29(1):61-84. doi:10.1200/JOP.2015.009407

8. Tomlinson JS, Jarnagin WR, DeMatteo RP, Fong Y, Kornprat $\mathrm{P}$, Gonen $\mathrm{M}$, et al. Actual 10-year survival after resection of colorectal liver metastases defines cure. J Clin Oncol Off J Am Soc Clin Oncol. 2007;25(29):4575-80. doi:10.1200/JCO.2007.11.0833

9. Bekaii-Saab T, Wu C. Seeing the forest through the trees: a systematic review of the safety and efficacy of combination chemotherapies used in the treatment of metastatic colorectal cancer. Crit Rev Oncol Hematol. 2014;91(1):934. doi:10.1016/j.critrevonc.2014.01.001

10. Adam R, Delvart V, Pascal G, Valeanu A, Castaing D, Azoulay D, et al. Rescue surgery for unresectable colorectal liver metastases downstaged by chemotherapy: a model to predict long-term survival. Ann Surg. 2004;240(4):64458. doi:10.1097/01.sla.0000141198.92114.f6

11. Hellman S, Weichselbaum RR. Importance of local control in an era of systemic therapy. Nat Clin Pract Oncol. 2005;2(2):60-1. doi:10.1038/ncponc0075

12. Borgelt BB, Gelber R, Brady LW, Griffin T, Hendrickson FR. The palliation of hepatic metastases: results of the radiation therapy oncology group pilot study. Int J Radiat Oncol Biol Phys. 1981;7(5):587-91. doi:10.1016/03603016(81)90370-9

13. Soliman H, Ringash J, Jiang H, Singh K, Kim J, Dinniwell $\mathrm{R}$, et al. Phase II trial of palliative radiotherapy for hepatocellular carcinoma and liver metastases. J Clin Oncol Off J Am Soc Clin Oncol. 2013;31(31):3980-6. doi:10.1200/ JCO.2013.49.9202

14. Pan CC, Kavanagh BD, Dawson LA, Li XA, Das SK, Miften $\mathrm{M}$, et al. Radiation associated liver injury. Int J Radiat Oncol Biol Phys. 2010;76(3 Suppl):S94-100. doi:10.1016/j. ijrobp.2009.06.092 
15. Lawrence TS, Robertson JM, Anscher MS, Jirtle RL, Ensminger WD, Fajardo LF. Hepatic toxicity resulting from cancer treatment. Int J Radiat Oncol Biol Phys. 1995;31(5):1237-48. doi:10.1016/0360-3016(94)00418-K

16. Dawson LA, Normolle D, Balter JM, McGinn CJ, Lawrence TS, Ten Haken RK. Analysis of radiation-induced liver disease using the Lyman NTCP model. Int J Radiat Oncol Biol Phys. 2002;53(4):810-21. doi:10.1016/s03603016(02)02846-8

17. Kini VR, Vedam SS, Keall PJ, Patil S, Chen C, Mohan R. Patient training in respiratory-gated radiotherapy. Med Dosim off J am Assoc. Med Dosim. 2003;28(1):7-11. doi:10.1016/S0958-3947(02)00136-X

18. Brock KK, McShan DL, Ten Haken RK, Hollister SJ, Dawson LA, Balter JM. Inclusion of organ deformation in dose calculations. Med Phys. 2003;30(3):290-5. doi:10.1118/1.1539039

19. Dawson LA, Balter JM. Interventions to reduce organ motion effects in radiation delivery. Semin Radiat Oncol. 2004;14(1):76-80. doi:10.1053/j.semradonc.2003.10.010

20. Dawson LA, Brock KK, Kazanjian S, Fitch D, McGinn CJ, Lawrence TS, et al. The reproducibility of organ position using active breathing control (ABC) during liver radiotherapy. Int J Radiat Oncol Biol Phys. 2001;51(5):1410-21. doi:10.1016/s0360-3016(01)02653-0

21. Hara W, Soltys SG, Gibbs IC. CyberKnife robotic radiosurgery system for tumor treatment. Expert Rev Anticancer Ther. 2007;7(11):1507-15. doi:10.1586/14737140. 7.11.1507

22. Wunderink W, Méndez Romero A, de Kruijf W, de Boer H, Levendag P, Heijmen B. Reduction of respiratory liver tumor motion by abdominal compression in stereotactic body frame, analyzed by tracking fiducial markers implanted in liver. Int J Radiat Oncol Biol Phys. 2008;71(3):907-15. doi:10.1016/j.ijrobp.2008.03.010

23. Case RB, Sonke JJ, Moseley DJ, Kim J, Brock KK, Dawson LA. Inter- and intrafraction variability in liver position in non-breath-hold stereotactic body radiotherapy. Int J Radiat Oncol Biol Phys. 2009;75(1):302-8. doi:10.1016/j. ijrobp.2009.03.058

24. Benedict SH, Yenice KM, Followill D, Galvin JM, Hinson W, Kavanagh B, et al. Stereotactic body radiation therapy: the report of AAPM Task Group 101. Med Phys. 2010;37(8):4078-101. [published correction appears in
Med Phys 2012;39(1):563. Dosage error in article text] doi: $10.1118 / 1.3438081$

25. Eisenhauer EA, Therasse P, Bogaerts J, Schwartz LH, Sargent D, Ford R, et al. New response evaluation criteria in solid tumours: revised RECIST guideline (version 1.1). Eur J Cancer. 2009; 45(2):228-47. doi:10.1016/j. ejca.2008.10.026

26. Rusthoven KE, Kavanagh BD, Cardenes H, Stieber VW, Burri SH, Feigenberg SJ, et al. Multi-institutional phase I/ II trial of stereotactic body radiation therapy for liver metastases. J Clin Oncol. 2009;27(10):1572-8. doi:10.1200/ JCO.2008.19.6329

27. Lee MT, Kim JJ, Dinniwell R, Brierley J, Lockwood G, Wong R, et al. Phase I study of individualized stereotactic body radiotherapy of liver metastases. J Clin Oncol. 2009;27(10):1585-91. doi:10.1200/JCO.2008.20.0600

28. Nicosia L, Cuccia F, Mazzola R, Figlia V, Giaj-Levra N, Riccheti F, et al. Stereotactic Body Radiotherapy (SBRT) Can Delay Polymetastatic Conversion in Patients Affected by Liver Oligometastases. J Cancer Res Clin Oncol. 2020;10.1007/s00432-020-03223-9. [Online, ahead of print].

29. Andratschke N, Alheid H, Allgäuer M, Becker G, Blanck O, Boda-Heggemann J, et al. The SBRT database initiative of the German Society for Radiation Oncology (DEGRO): patterns of care and outcome analysis of stereotactic body radiotherapy (SBRT) for liver oligometastases in 474 patients with 623 metastases. BMC Cancer. 2018;18(1):283. doi:10.1186/s12885-018-4191-2

30. Mahadevan A, Blanck O, Lanciano R, Peddada A, Sundararaman S, D'Ambrosio D, et al. Stereotactic Body Radiotherapy (SBRT) for liver metastasis - clinical outcomes from the international multi-institutional RSSearch ${ }^{\circledR} \mathrm{Pa}-$ tient Registry. Radiat Oncol. 2018;13(1):26. doi:10.1186/ s13014-018-0969-2

31. Scorsetti M, Arcangeli S, Tozzi A, Comito T, Alongi F, Navarria $\mathrm{P}$, et al. Is stereotactic body radiation therapy an attractive option for unresectable liver metastases? A preliminary report from a phase 2 trial. Int J Radiat Oncol Biol Phys. 2013;86(2):336-42. doi:10.1016/j.ijrobp.2012.12.021

32. Rule W, Timmerman R, Tong L, Abdulrahman R, Meyer J, Boike T, et al. Phase I dose-escalation study of stereotactic body radiotherapy in patients with hepatic metastases. Ann Surg Oncol. 2011;18(4):1081-7. doi:10.1245/s10434010-1405-5 